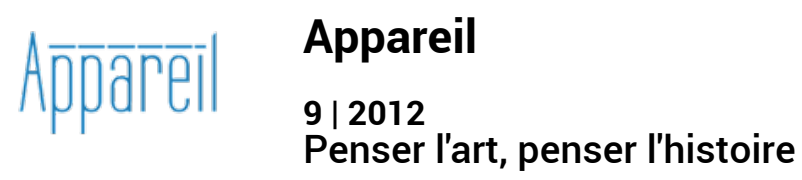

\title{
Carnation et incarnation
}

Logique picturale et logique religieuse dans l'œuvre tardive du Titien

\section{Thomas Kisser}

Traducteur : Audrey Rieber

\section{OpenEdition}

\section{Journals}

Édition électronique

URL : http://journals.openedition.org/appareil/1465

DOI : 10.4000/appareil. 1465

ISSN : 2101-0714

\section{Éditeur}

MSH Paris Nord

Référence électronique

Thomas Kisser, «Carnation et incarnation », Appareil [En ligne], 9 | 2012, mis en ligne le 05 juillet 2012, consulté le 30 juillet 2020. URL : http://journals.openedition.org/appareil/1465 ; DOI : https://doi.org/ 10.4000/appareil.1465

Ce document a été généré automatiquement le 30 juillet 2020.

\section{(c) (i) (2) $\Theta$}

Appareil est mis à disposition selon les termes de la Licence Creative Commons Attribution - Pas d'Utilisation Commerciale - Pas de Modification 4.0 International. 


\title{
Carnation et incarnation
}

\author{
Logique picturale et logique religieuse dans l'œuvre tardive du Titien
}

\author{
Thomas Kisser
}

Traduction : Audrey Rieber

\footnotetext{
«La chair est le véritable Chaos de toutes les couleurs et pour cela ne ressemble à aucune mais est leur mélange le plus indissoluble et le plus beau. De plus, cette espèce bien particulière de couleur n'est pas immobile comme les autres espèces de couleur, mais vivante et mobile ${ }^{1}$ ".
}

1 À l'époque de la Renaissance et de la Réforme, l'art a conquis une autonomie toujours croissante qui s'est traduite par le prestige social du peintre, l'apparition d'un marché de l'art ainsi que par le mode d'élaboration des images. La recherche de l'originalité ainsi qu'une interprétation spécifiquement esthétique de l'art témoignent d'une dynamique artistique immanente qui se développe indépendamment d'autres fonctions sociales. $\mathrm{Si}$, dans ses grandes lignes, ce processus d'autonomisation est unanimement reconnu, la question se pose néanmoins de savoir comment il opère dans le détail. À partir d'une œuvre tardive du Titien, Le Couronnement d'épines de Munich (Ancienne Pinacothèque), j'essayerai de montrer comment cette autonomisation modifie les rapports entre l'art et la religion et de quelle façon elle entraîne une modification du concept d'œuvre.

2 L'histoire sainte, la théologie chrétienne attribue les actions d'une manière bien déterminée. Tandis que l'acte propre à l'homme est le péché, celui de Dieu est la Grâce. Entre les deux se déploie la libre volonté de l'homme qui participe également de la grâce divine et du péché humain. On sait que c'est cette question et son interprétation qui donneront lieu au schisme confessionnel du siècle de Titien. Le paradoxe chrétien d'une rédemption dans un monde qui de toute évidence n'est pas racheté s'aiguise et le problème de l'attribution (des actions) se fait toujours plus urgent. La justice des œuvres ne limite-elle pas le caractère nécessaire de la grâce ? Inversement, Dieu peut-il vraiment commander et permettre l'aveuglement? Ou encore : le concept luthérien de foi, qui conditionne dans une certaine mesure la grâce, parvient-il à lever 
définitivement le paradoxe ? Historiquement, le conflit d'attribution, d'abord interne à la théologie, témoigne d'une remise en question de la capacité intégratrice de la religion; finalement, la religion elle-même se voit attribuée, devenant relative, et de nombreuses attributions dans la société se retrouvent indéterminées et en crise $^{2}$. La notion artistique d'œuvre s'inscrit elle aussi dans ces débats. Qui peint? La peinture d'icônes permet de se faire aujourd'hui encore une idée des difficultés rencontrées par l'art occidental pour se dégager de la juridiction religieuse.

À l'instar du témoin - un martyre, un saint - qui, bien qu'il parle, ne témoigne pourtant pas de lui-même mais du Seigneur et fait apparaître non lui-même mais Lui, les peintres d'icônes témoignent non de leur art de peindre des icônes, c'est-àdire d'eux-mêmes, mais des saints, les témoins du Seigneur et, par eux, du Seigneur lui-même ${ }^{3}$.

L'activité artistique se soucie donc de transparence et l'on peut dire que l'agir du peintre lui-même se dissout en quelque sorte dans la vue de Dieu.

4 Je me propose d'illustrer l'évolution du concept d'œuvre en comparant et analysant les représentations du couronnement d'épines par le Titien. Ce moment culminant de l'histoire sainte, qui exprime le paradoxe du règne du Christ sur le monde à travers les moqueries de ses tortionnaires, a été peint à deux reprises par Titien dans un format et une organisation presque identiques. La première version, aujourd'hui au Louvre, a été conçue vers 1540 (figure 1) pour l'église Marie delle Grazie de Milan qui possède la relique d'une épine de la Sainte Couronne ; la seconde version, conservée à l'Ancienne Pinacothèque de Munich (figure 2), est l'une des dernières images peintes par le Titien vers $1570^{4}$. Trente ans séparent les deux images. Comment se fait-il qu'en dépit d'un tel intervalle de temps elles présentent une ressemblance bluffante? Les examens radiographiques ont montré que le Titien a peint la seconde image sur une copie de la première ${ }^{5}$. 
Figure 1

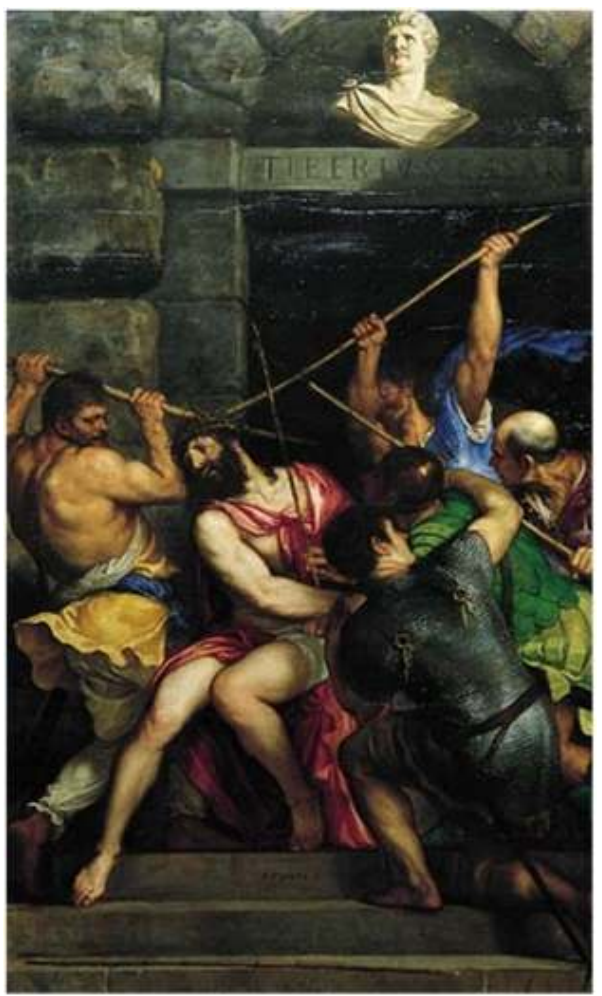

Le couronnement d'épines, Titien, vers 1542-1543, huile sur toile ; dimensions : 303 × 180 cm, Paris, Musée du Louvre. 
Figure 2

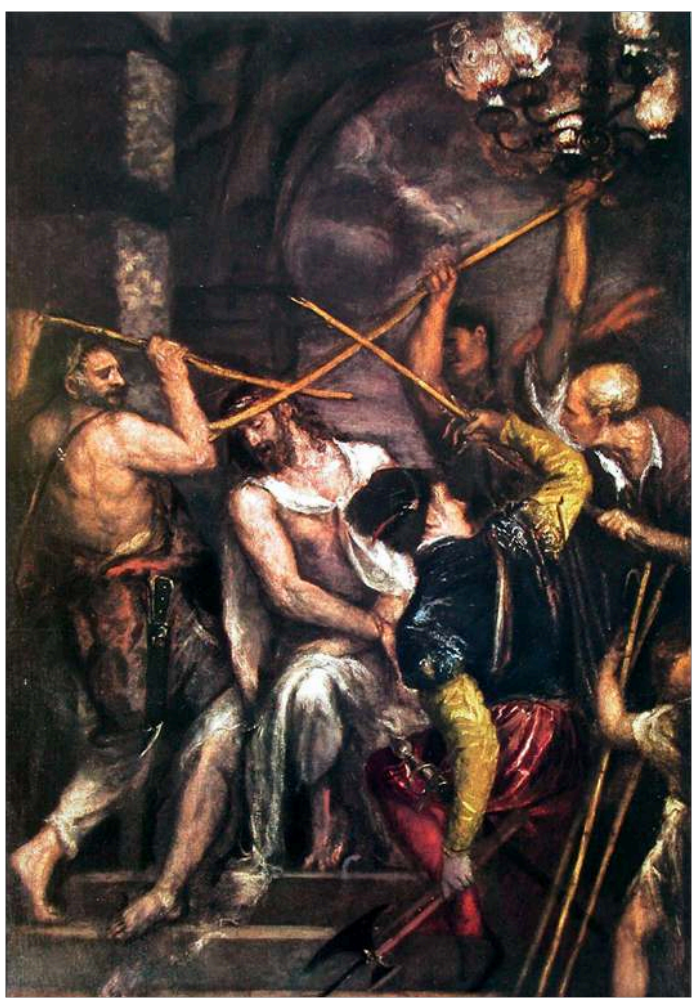

Le Couronnement d'épines, Titien, vers 1572, huile sur toile ; dimensions : 280 x 181 cm, Munich, Alte Pinakothek.

Les expertises radiographiques dévoilent au premier regard la modification dramatique que le Titien a faite subir à l'image initiale qu'il avait sous les yeux alors qu'il la repeignait. Cette première image présente des figures clairement délimitées, douées d'une haute valeur plastique. Les historiens d'art ont vu leur modèle dans la sculpture antique (voyez par exemple la figure du sbire de gauche) et ont reconnu dans la figure de Jésus des réminiscences du Laocoon ${ }^{6}$. L'empereur Tibère, sous le règne duquel Jésus fut crucifié, est présent sous la forme d'un buste de style antique. Les corps sont ordonnés selon un mouvement circulaire ou tournant qui part du soldat situé en bas à droite, se poursuit vers le haut avec deux autres figures, puis redescend jusqu'au milieu de l'image avec le mouvement du quatrième affidé et de son bâton. Le mouvement descendant du quatrième agresseur et de sa verge est encadré et renforcé par la porte quadrangulaire et l'arc en plein cintre qui la surplombe. La rotation du corps et du bâton de l'agresseur de gauche dirige ce mouvement vers Jésus qui constitue le centre douloureux mais aussi résistant de l'action et de la composition. Bien que cette composition d'ensemble monumentale mette les personnages en rapport les uns avec les autres, aucun n'y perd son autonomie. Tous les mouvements sont effectués par des corps individuels traités comme des centres d'action et même lorsque le mouvement est contraint, comme c'est le cas chez Jésus, on voit le gonflement des muscles et la résistance du corps. Comme les figures, le mur fait un effet solide et monumental. En toute logique, les couleurs de l'image sont des propriétés de ces corps, c'est-à-dire des choses comme les vêtements et le mur. Les couleurs forment également une composition d'ensemble, mais leurs contrastes demeurent accusés. Elles ne sont pas mélangées, chaque figure ayant une teinte propre. 
Considérons à présent la version de l'Ancienne Pinacothèque de Munich, de trente ans postérieure. L'ordonnancement des figures correspond dans l'ensemble à la première version. Mais contrairement à elle, les objets individuels ne sont plus clairement délimités. L'image produit d'abord un effet coloré très homogène et ce n'est que peu à peu que des impressions individuelles se détachent toujours plus nettement. Ce phénomène n'est malheureusement observable que devant l'image, car l'excessive netteté de la plupart des reproductions photographiques nous épargne ce travail de l'œil, si important pour les œuvres tardives du Titien. C'est seulement devant l'original qu'on reconnaît petit à petit des contrastes toujours plus nombreux et qu'on découvre par degrés la bigarrure tout simplement fantastique de l'image. Il en demeure une teinte générale, une certaine qualité fondamentale homogène. Celle-ci s'avère être la couleur de la peau qui varie aussi bien dans les corps que dans les choses où elle tend vers le brun terreux. La carnation y est modifiée par les objets et par le rapport à la lumière. Celle-ci ne vient pas seulement, tel un rayon, directement de la source lumineuse (le flambeau à cinq branches, en haut à droite), mais est aussi présente en tant que modification des couleurs. La façon de peindre est irrégulière : la couleur est tantôt appliquée très minutieusement tantôt jetée comme une tache sur la toile?

7 Mais examinons plus précisément la construction de l'image. L'ordonnancement des personnages est quasiment le même que dans la première version, et le soldat vu de dos y est pareillement le point de départ du mouvement de la composition et du parcours du regard. Parce que nous nous tenons au niveau du bord inférieur du tableau et donc devant les marches, notre regard pénètre dans l'image à travers la figure vue de dos, est dirigé vers le haut par le mouvement ascendant de son bras droit puis, au travers de l'agresseur qui se tient derrière, est à nouveau orienté, tourné pourrait-on dire, vers le bas $^{8}$. Les lances montrent très clairement ce mouvement. Le regard suit d'abord la hallebarde que la figure de dos tient de la main gauche et qui, partant de l'axe médian, indique la partie supérieure droite du tableau. Il considère ensuite les lances qui se dressent verticalement dans les bras de l'enfant placé à droite, tout en bas de l'image, puis, enfin, le bâton que la figure de dos brandit de sa main droite et qui, recouvrant celui de l'autre soldat, est exactement placé à la surface. Ces deux bâtons opèrent un changement de direction du côté supérieur gauche vers l'axe médian de l'image. Cette ligne rencontre le bâton du troisième soldat qui, partant du côté supérieur droit, engendre un mouvement du haut vers le milieu du tableau. Ce mouvement est encadré par l'arrondi de l'arc du portail au fond, le linteau horizontal de l'image du Louvre ayant disparu. Le pilier droit n'est pas visible - comme dans la première version - et l'arc gauche se dirige vers l'épaule de Jésus. À la différence du tableau de Paris, les lances accentuent généralement la surface aux dépens de l'espace, un effet que renforcent leurs positions peu vraisemblables. Sous l'arc de la porte enfin, la surface peinte de l'image s'ouvre entièrement sur un arrière-fond insondable et irreprésentable qui, certes se laissait déjà deviner dans la version antérieure, mais qui donne ici forme à un événement presque métaphysique.

8 La position du corps de Jésus et celle du soldat de gauche capturent les mouvements de rotation descendants et légèrement dirigés vers la gauche; avec la lance qui croise les autres, ils calment le mouvement du regard qu'ils amènent au centre de l'image. Ce faisant, le corps de Jésus est dévissé de ou vissé dans l'arrière-fond de l'arc, un effet accentué par la rotation du sbire au milieu de l'image. La rotation de la figure christique la relie aussi bien au fond sombre ouvert de l'arc du portail qu'au flambeau 
qui l'éclaire ; la lumière de la torche et la profondeur ouverte du fond s'unissent donc en Jésus et centrent l'image. La lumière laisse ainsi le regard descendre le long du corps du Christ vers nous, les spectateurs, une direction qui est surtout explicitée par le genou gauche du Christ. Le corps de Jésus qui a endossé la souffrance centre donc le mouvement de l'image.

9 Le traitement des corps dans la seconde version du tableau diffère donc très nettement de celui de la première. Tous les corps, et pas seulement celui de Jésus, ont perdu l'essentiel de leur tension et de leur mouvement autonome. En dépit des positions qu'ils prennent et de leurs mouvements pour ainsi dire vigoureux - qu'on observe par exemple le soldat au manteau flottant, en haut à droite-, les corps semblent immobilisés et privés de mouvement propre ${ }^{9}$. Le mur aussi a perdu sa massivité et semble presque flotter avec sa pierre supérieure qui le fait comme basculer vers l'avant. Privés de leur pouvoir, les corps doivent de toute évidence obéir à une structure supérieure ${ }^{10}$.

10 Considérons à présent les couleurs. Leur modification suit le mouvement du soldat de droite vers Jésus. Seul cet homme vu de dos, qui plie le genou, est habillé : il porte une culotte, une chemise, par-dessus un pourpoint sans manches, et une toque. Les couleurs du tableau ne sont pas traitées de manière synthétique comme expression d'un ton dominant homogène, mais sont présentées pour elles-mêmes et si l'on veut analytiquement: rouge, noir, jaune et or. Dans les autres parties de l'image, les couleurs sont mélangées; quant à la figure intensément blanche de Jésus, doublement tournée vers la source lumineuse et le fond sombre, elle tend à réduire la couleur au clair et au foncé. Un mouvement de rotation permet donc de faire passer d'une représentation analytique des couleurs, dans le vêtement de la figure de dos, à leur combinaison synthétique, qui ouvre un champ de contrastes en permanent changement, et à la composition claire et sombre très peu colorée du centre où s'origine en quelque sorte le coloris. À la différence de la première version, le coloris se distingue de la composition figurative qu'il chevauche, l'emportant donc visuellement sur elle. Ce sont les intensités colorées qui déterminent l'image. Il en résulte que toute la composition et le parcours du regard, si proches soient-ils de ceux de la première version, sont constamment relativisés, y compris lors une contemplation prolongée.

11 L'autre extrémité de la coloration est constituée par Jésus, c'est-à-dire par le clair et foncé qui se dissout dans l'obscurité du fond et la lumière du flambeau d'où il devient productif. Pour représenter le monde comme synthèse de la carnation, le peintre se réfère donc au Dieu porteur de lumière et devenu chair, par lequel seul le monde existe et se voit sauvé, ramené à la sainteté. L'acte créateur du peintre, une nouvelle fois rendu visible, se représente derechef dans le mouvement des couleurs analytiques vers le centre de l'image ; il rend ainsi manifeste un acte créateur antérieur, l'incarnation, et répète dans la couleur l'acte divin. «Et vraiment, c'est consciemment qu'il travaillait de la sorte. Il voulait imiter l'activité du plus grand des créateurs et modelait de ses mains le corps humain avec de la terre ${ }^{11}$ ».

12 La coloration fait donc de Jésus et de la figure de dos deux pôles qui, d'emblée, se limitent l'un l'autre à la surface de l'image. La représentation analytique de la couleur se tient au seuil de l'image, du côté du spectateur mais également du peintre. En ce sens, l'acte de peindre est encore une fois représenté, car le peintre crée à partir des couleurs individuelles la surface complexe sur laquelle la lumière se figure dans la chair. C'est comme si la figure de dos nous donnait à voir la palette du peintre : le 
rapport entre analyse et synthèse permet donc de représenter l'acte créateur luimême. Très concrètement, on peut refaire l'expérience de cet acte créateur lorsqu'on examine l'image à des distances différentes. Vue de près, en effet, la texture de la surface, véritable produit de l'acte de peindre du Titien, n'est pas organisée mais presque uniquement constituée de taches de couleurs. Elle se distingue donc structurellement de l'impression de formes que l'on éprouve à une certaine distance. C'est le Titien qui introduisit pour la première fois cet effet dans la peinture, ce dont Vasari, le théoricien du disegno, s'irrita fort ${ }^{12}$.

D'autres éléments encore vont nous permettre de confirmer et d'éclairer l'emploi conscient que le Titien fait de la carnation. Considérons sa Jeune fille avec plateau de fruits conservée à Berlin (figure 3$)^{13}$ qui présente elle aussi une modification immanente de la carnation. La carnation y apparaît un peu plus bariolée et pure, avec des tons jaunes et or accentués, mais on y retrouve la même séparation entre synthèse et analyse. Les couleurs synthétiques de l'image, comprises comme modifications de la carnation, sont répétées analytiquement dans le plateau de fruits. Aux côtés du bleu du métal et du rouge de la pomme, le jaune est significatif et ressemble beaucoup au ruban de même couleur qui parcourt le dos du soldat de Munich. Panofsky voit dans la fillette une antique déesse de la fécondité ${ }^{14}$; elle serait donc en quelque sorte le pendant païen du couronnement d'épines de Munich. Une troisième image, conservée au Prado cette fois, mérite encore être mentionnée ${ }^{15}$. On y voit, dans la même attitude exactement, une Salomé portant une coupe avec, cette fois, la tête de saint Jean (figure 4). Ici, c'est la robe qui est traitée de manière analytique, le rouge, le bleu et le blanc étant clairement distingués dans les différentes parties de l'habit. La tête de Jean en revanche possède le caractère synthétique de la couleur chair. Nous avons caractérisé de palette la représentation analytique, individuelle, des couleurs. Qu'il ne s'agisse pas d'une pure métaphore, d'une métaphore propre au Titien, c'est ce que confirme un tableau de Dresde. À l'arrière-plan de cette image peinte en 1561 (figure 5), s'ouvre à gauche un paysage; on y passe d'une terre brunâtre et d'un arbre virant au rouge à un ciel jaunâtre puis bleu et, enfin, à des tons verts en haut. Chacune des couleurs se trouve isolément dans une boîte à peintures placée juste sous l'embrasure de la fenêtre ; un couteau de peintre est posé sur cette boîte, comme si la nature croissait à partir d'elle. Ce tableau, dans lequel on a longtemps vu un portait d'Antonio Palma, dit Palma le Jeune, représenterait selon les recherches les plus récentes le marchand de peinture Alvise dalla Scala, surnommé dai colore ${ }^{16}$. Le portrait démontre littéralement la signification matérielle, mieux : formelle de l'acte créateur. 
Figure 3

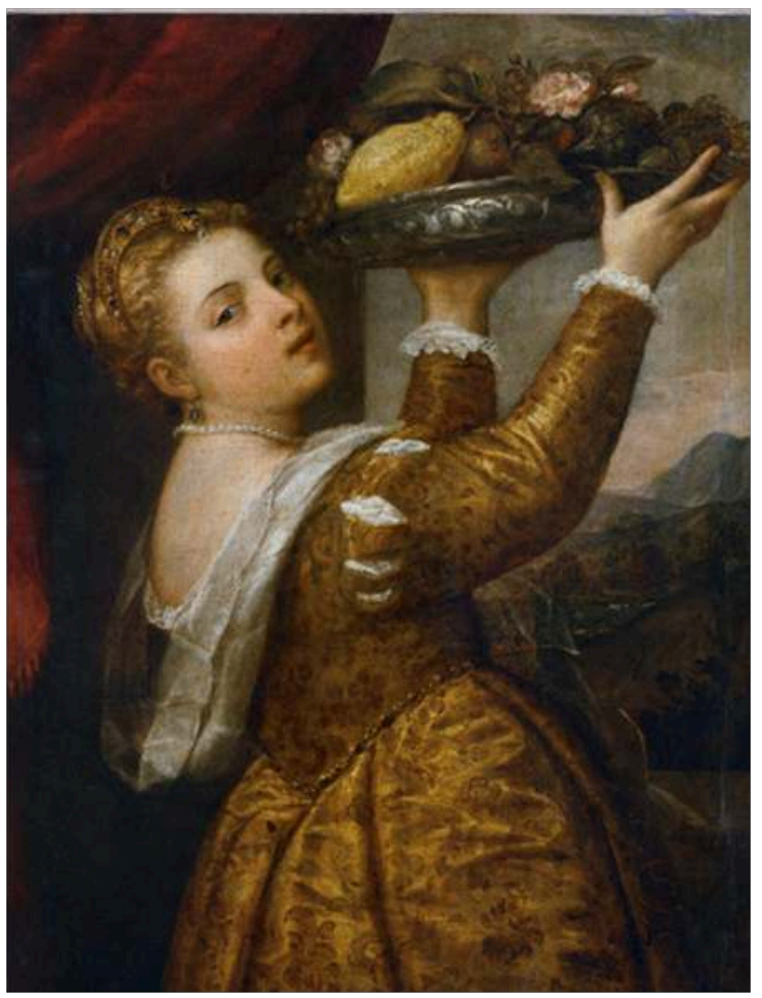

Jeune fille avec plateau de fruits, Titien, vers 1555, huile sur toile, 102 × $82 \mathrm{~cm}$, Berlin, Gemäldegalerie.

Figure 4

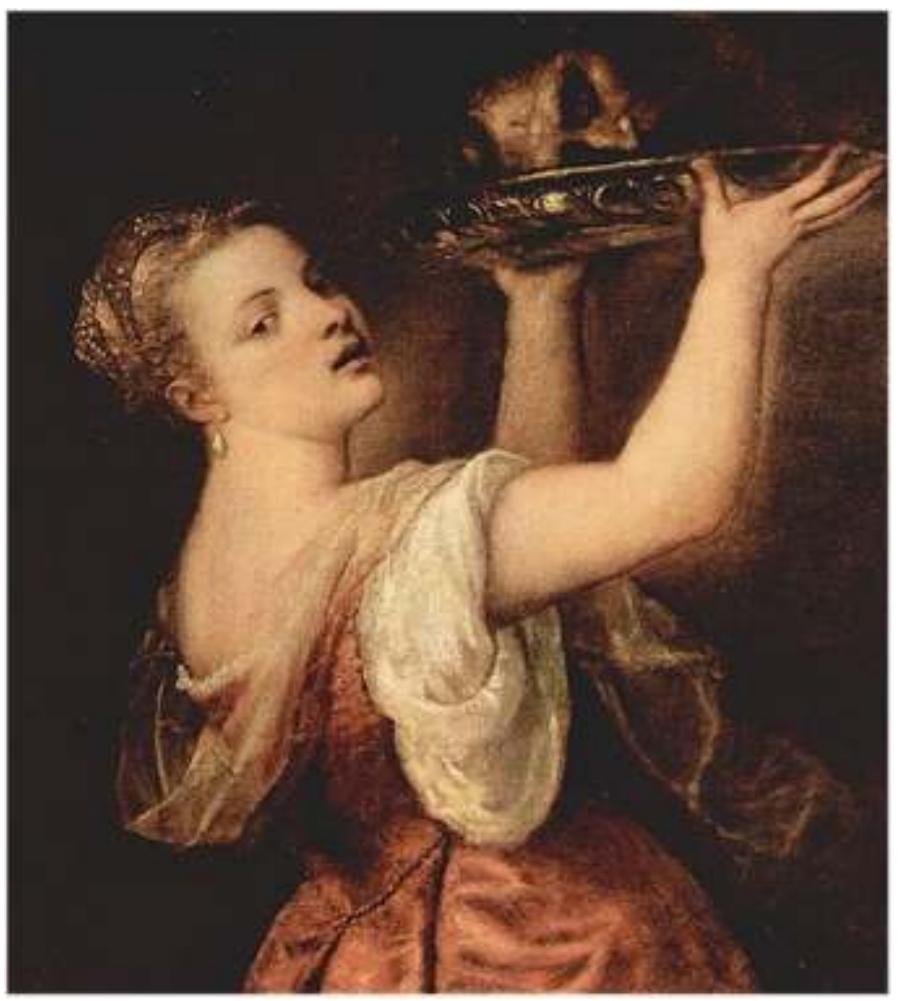

Salomé portant la tête de saint Jean-Baptiste, Titien, huile sur toile, 80 × $87 \mathrm{~cm}$, Madrid, Musée du Prado. 
Figure 5

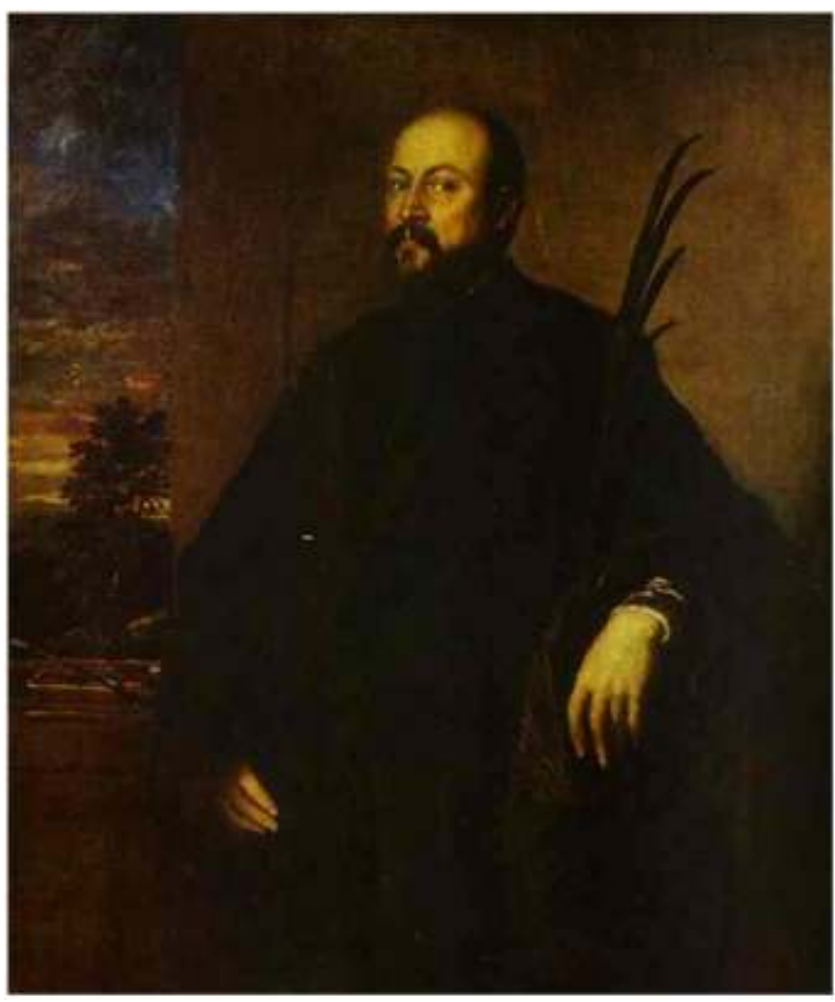

Portrait d'homme à la palme, Titien, 1561, 116 x 138 cm, Dresde, Gemäldegalerie s'emparer souverainement de la composition. Cette évolution du Titien doit être appréhendée dans un cadre plus vaste qui, depuis 1500, marque la peinture occidentale. « La surface devient une entité ornementale, n'est plus la parete di vetro (la paroi d'une vitre). Il n'y a plus de scène, d'espace conçu comme une boîte dans lesquels les figures sont pour ainsi dire placées après coup, l'image est bien plutôt développée selon les possibilités de la division de la surface, en rapport exact avec le cadre ${ }^{17}$ ». C'est pourquoi « chaque forme [...] est dans un rapport non pas simplement figuratif mais vraiment essentiel avec les autres ${ }^{18}$ ». C'est en ces termes que Theodor Hetzer caractérise les changements dans la conception de l'image au début du $\mathrm{xvI}^{\mathrm{e}}$ siècle. L'espace et les figures n'ont plus un rapport extérieur entre eux, comme si l'on pouvait découper les figures dans l'image sans que la conception de l'espace disparût. Les figures vivent dans les rapports de composition dont elles naissent ; quant à l'espace, il est construit en fonction des figures. À cette nouvelle conception de l'image appartiennent notamment la perspective aérienne ou l'usage de diagonales. Dans l'œuvre de Titien, que Hetzer caractérise de façon cruciale d' " histoire de [la] couleur ", ce développement, déjà repérable dans la première version du Couronnement d'épines comme agencement organique des corps, sera, dans les dernières années, surtout poursuivi avec la coloristique. Titien développe une conception radicalement neuve de la couleur. Dans la première version du Couronnement d'épines mais aussi dans la pratique quotidienne de la perception, la couleur est d'abord considérée comme une propriété des choses. Or ce rapport s'inverse désormais, la conception des couleurs étant immédiatement corrélée à celle des objets. «En tant que matière du tableau », la 
couleur « est organisée de manière analogue à la substance de la nature ${ }^{19}$ ». Les objets procèdent désormais de la couleur; elle n'est donc plus une propriété des choses mais devient la « parabole par excellence d'une substance, de la matière remplie par l'action divine. La matière est la substance d'où tout procède et dans laquelle tout se défait à nouveau ${ }^{20} »$. Cette analyse peut être mise en rapport avec la structure de la version tardive du Couronnement d'épines dans laquelle on peut reconnaître comme trois moments de la matérialité : l'émergence de la substance colorée comme substance du monde à partir du clair-obscur, son action comme médium et substance du monde et enfin sa décomposition analytique qui, derechef, est le point de départ de l'acte de peindre. Ainsi comprise, la matière ne s'oppose pas simplement à la forme mais est la substance vivante elle-même qui vit d'elle-même et s'autodifférencie, forgeant les formes à partir et en elle-même. Les formes sont donc des formes immanentes de la matière ; on pourrait dire que la matière devient une forme générale qui façonne à partir d'elle-même et en elle-même les formations individuelles. Dans la mesure où la couleur qui déforme le corps concret domine la composition figurative, elle pose les déterminations individuelles des moments du tout dont elles procèdent et au sein duquel elles se trouvent dans le flottement d'une indétermination, la relativisation de toute individualité solide.

Le traitement du tableau comme surface et champ, la perte de pouvoir des figures individuelles et l'élaboration de la composition comme différenciation d'une qualité colorée fondamentale forment un clair parallèle avec le développement contemporain de la conceptualité philosophique et la compréhension de soi de la modernité qui s'y révèle. La dynamique du développement philosophique moderne réside pour l'essentiel dans la désubstantialisation du monde. Successivement élaborée dans les systèmes métaphysiques du Cusain à Leibniz, elle n'a cessé de progresser depuis la fin du Moyen Âge. Vue négativement, elle se manifeste d'abord sous l'espèce d'une critique nominaliste de la conception aristotélicienne traditionnelle de l'être comme substance, comme composition d'une forme formante et d'une matière passive et sans forme. Vue positivement, le monde comme champ se déplace dans le regard où plus aucune individualité ne peut être comprise indépendamment et pour elle-même, mais existe seulement comme moment d'un continuum, comme position dans un champ. L'individu n'est plus compris par lui-même et en tant tel, mais uniquement comme partie d'une effectivité qui l'englobe ${ }^{21}$. Chaque individu n'est ce qu'il est que par sa position dans le tout, dans sa relationalité aux autres. La relationalité n'est donc ni extérieure ni donnée après coup: elle est la genèse propre de l'individu. Pour Aristote, les choses individuelles comme substances ont en elles-mêmes leur effectivité comprise comme fin. Ce telos transfère la dynamique entièrement immanente de la forme formante à une temporalité en fin de compte inessentielle qui est due à la seule absence de forme de la matière et qui n'a de valeur qu'au sein du monde sublunaire dans lequel, justement, les formes dépendent d'une matière. La relation en tant que telle tout comme les déterminations catégoriales de l'espace et du temps deviennent donc de simples accidents, des accessoires seulement extérieurs de l'être véritable des substances. En toute rigueur, on ne peut pas parler d'espace chez Aristote. Le concept aristotélicien de lieu, âprement critiqué au début de l'époque moderne, considère l'espace de la substance individuelle comme son espace. La substance individuelle, conçue comme l'être véritable, est déterminée par les formes de l'être que nous percevons de nouveau à partir de la substance individuelle avant de les réaliser comme formes dans la mémoire abstractive et l'entendement actif. L'initiative vient ce faisant de la forme de 
la chose singulière, de ce qui est véritablement réel dans le monde, avant de s'unir avec la forme du spectateur, être rationnel, avec la raison active, partie la plus élevée de l'âme, dans la connaissance de la forme de l'être ${ }^{22}$. En adoptant ce que Panofsky a nommé la perspective corporelle - qui consiste à peindre les corps dans une sorte d'espace propre qui ne compose pas une spatialité unifiée avec l'espace des autres corps-, la peinture antique suit en quelque manière ce principe plastique substantialiste ${ }^{23}$. Mais c'est surtout la sculpture antique qui, en faisant du contrapposto une forme paradigmatique, montre cette substantialité, ce pouvoir-être-pour-soi. Et nous avons vu comment, dans la première version du Couronnement d'épines, le Titien se rattachait encore à cette image du corps substantialiste et plastique qui définit aussi par exemple la peinture de Mantegna.

16 Les théories antiques de la vision et de la pensée adoptent elles aussi ce substantialisme ${ }^{24}$. La perception, puissance passive activée par les formes de l'être et, dès le départ donc, codée par ces formes, est au fondement de la compréhension de l'art. Pour Aristote, l'art est donc toujours une représentation d'actions humaines ${ }^{25}$. Mais parce que par principe les actions des hommes suivent le telos et réalisent la vertu, l'art aussi représente avant tout l'accomplissement ou l'échec fatal d'une fin. La subordination de la représentation artistique à la structure de l'action et de la vertu humaines a marqué la tradition picturale jusque tard dans la modernité, sous la forme du tableau d'histoire moderne.

17 Ce renversement que constituent la perte de substance du monde et la détermination nouvelle de l'individuel s'amorce avec le concept d'univocité de l'être du théologien franciscain Jean Duns Scot selon lequel tous les êtres participent à un seul et même être. En réaction au nominalisme d'Occam qui comprend le monde comme une accumulation d'objets irréductiblement individuels dont il minimise et pragmatise le cadre catégorial ${ }^{26}$, le scotisme conçoit, lui aussi à partir d'un intérêt théologique pour l'individuel, le monde comme un ensemble essentiellement un qui se différencie en individus. Le point de départ de cette thèse est le concept aristotélicien de qualité comprise comme accident, exemplairement illustrée par l'être-blanc. Tandis qu'il est essentiel pour Socrate d'être un homme, c'est-à-dire d'être une nature ou une forme au sens essentiel, il n'est en revanche pas essentiel pour l'homme d'être blanc ou noir ou d'être de quelque couleur que ce soit. Le scotisme fait de cette nature seconde, ou qualité, le paradigme d'une nouvelle compréhension de l'effectivité. Il conçoit l'individu comme un degré déterminé de la qualité en elle-même une de l'être, de l'ens inquantum ens, objet premier de la métaphysique, tout comme il fait de tout blanc visible un degré déterminé du blanc. L'effectivité, qui résulte de l'unité univoque de la détermination de forme ainsi que des différences qui deviennent possibles, c'est-à-dire effectives, au sein de cette détermination formelle, constitue donc un champ. Les différences ne sont pas essentielles mais graduelles; la seule et unique différence est celle entre fini et infini et tous les individus en général participent de la même forme. Les individus ne sont donc plus les instanciations d'une même forme d'être, mais plutôt des singularités dans un champ ${ }^{27}$. Par sa finitude, chaque fini indique donc l'infini, l'unité sans limites qui se représente elle-même dans le fini. La détermination de la forme n'est plus visible, elle n'est plus accessible à l'expérience concrète ou à la perception en tant que détermination catégoriale du champ ; elle ne peut dorénavant être que pensée et devient en ce sens virtuelle. Cette virtualité ouvre l'espace de la pensée a priori moderne comme scientia transcendens, une science qui va au-delà de l'effectivité expérimentable dont elle thématise la possibilitée ${ }^{28}$. Le champ de force 
comme détermination dynamique de l'effectivité, l'espace donc de la physique moderne, est lui aussi un tel ensemble ou système différentiel. Il a été formulé pour la première fois par les calculateurs d'Oxford, une école scolastique tardive influencée par le scotisme ${ }^{29}$. La proposition de Leibniz: «Il y a une infinité de degrés dans les monades » ne vaut pas que pour sa philosophie et montre la signification fondamentale de la graduation pour la métaphysique depuis le $\mathrm{xIV}^{\mathrm{e}}$ siècle $^{30}$. La gradualité permet de développer d'une manière inédite l'idée d'une multiplicité qui est toujours déjà en rapport avec l'unité.

C'est dans cette perspective qu'il faut comprendre la couleur du Titien. Celle-ci forme une qualité fondamentale homogène qui demeure en tant que telle virtuelle, mais qui se manifeste dans des singularités innombrables aux qualités différentes, traitées avec le plus grand soin ou traits apparemment sommaires. C'est ainsi qu'elle montre sa vie. Les individualités ou singularités se différencient non par la forme univoque, qui reste toujours la même, mais par la détermination concrète matérielle. La matière donc n'est plus quelque chose de passif qui, comparée à la forme, aurait besoin d'être formée, mais devient une forme ouverte, une matière vivante qui autorise et opère en elle-même des réalisations infinies. Le fond est désormais une virtualité infinie et la forme individuelle, une modification. Ce rapport entre la forme et la matière permet de penser les différentes déterminations non comme des unités hétérogènes de forme et de matière, mais comme une autodifférenciation, c'est-à-dire comme l'expression d'une substance primitive qui se modifie en singularités. C'est exactement ce rapport entre virtualité et actualisation que la coloristique du Titien rend visible.

Malgré leurs tentatives pour conceptualiser l'art de peindre du Titien, les historiens d'art n'évoquent pas cette ontologie qui semble ne leur être guère familière. Par son caractère d'événement où la matière comme forme vivante se différencie, cette ontologie de l'univocité nie l'universalité de la forme idéale. Elle s'oppose donc aux deux positions présentées par Valeska von Rosen et offre une conceptualité à même d'éclairer la pensée artistique du Titien et tout particulièrement ses images tardives. Dans sa thèse qui fait le bilan des recherches actuelles sur le Couronnement d'épines, Valeska von Rosen oppose la peinture du vieux Titien à la conception du monde substantialiste d'Aristote, pour lequel la couleur est un simple accident, ainsi qu'à la théorie du disegno au nom de laquelle Vasari a même critiqué le Titien ${ }^{31}$. Dans les faits, et il faut le souligner, ces deux versions du monde insistent également sur la clarté de la forme comprise comme contour et représentent - c'est ma contribution à cette question - un concept de forme pré-moderne basé à la fois sur l'autonomie de la forme individuelle et, pour le dire vite, sur la dévalorisation de la matière. Comme je l'ai montré, l'ontologie franciscaine de l'univocité inverse en quelque sorte les rapports et développe, à partir de la forme matérielle vivante, des singularités individuelles qui font disparaître aussi bien le dualisme aristotélicien entre forme et matière que «le platonisme où l'individu est une simple copie de l'image originelle ${ }^{32}$ ». Se pose bien sûr la question de l'influence directe d'une telle idée sur le Titien, c'est-à-dire sur la littérature artistique du temps. Même si cette doctrine est volontiers négligée par les thomistes, l'organisation en ordres de l'enseignement ne laisse aucun doute quant à sa présence dans toute l'Europe. À l'université de Padoue existait depuis 1450 une chaire scotiste qui concurrençait explicitement la thomiste, ce qui avait d'ailleurs favorisé la publicité des débats en la matière ${ }^{33}$. À partir de 1480 environ, on y lisait les traductions latines des commentateurs grecs d'Aristote qui, à l'instar du matérialiste Alexandre d'Aphrodise ou de l'inventeur de la théorie de l'impetus, Philiponos, vont bien dans le 
sens du concept d'univocité, même si la recherche, suivant en cela les philosophes de la Renaissance, a tendance à voir deux partis et à séparer les scolastiques Thomas et Scot, d'un côté, des nouveaux philosophes de la nature et des humanistes, de l'autre. L'aristotélisme padouan tout particulièrement cherche, en conformité avec la pensée scotiste, à séparer la philosophie de la nature de la théologie et à émanciper le concept de matière ${ }^{34}$.

20 L'ontologie de l'univocité engage aussi une nouvelle détermination de la négation. Contrairement au modèle aristotélicien de la forme et à l'idéal du disegno, la négation n'est plus comprise comme une privation, c'est-à-dire comme le manque d'une norme pré-donnée, mais comme une réaction à « une négativité en elle-même indéterminée qui dépend d'une négation déterminante", pour reprendre les termes par lesquels Niklas Luhmann caractérise l'ontologie moderne et qui correspondent tout à fait au processus de peinture du Titien qui travaille par limitations du fond sombre en nouvelles couches de couleurs ${ }^{35}$. Mais la relativisation décidée de la forme figurative des corps et des choses fait surgir dans l'image une dimension temporelle qui ne peut être comprise ni comme le souvenir d'une action déterminée (que l'on reconnaît essentiellement par la signification) ni comme son actualisation. Tout être apparait désormais dans son devenir et sa disparition, et cet apparaître et disparaître simultanés indiquent exactement la différence ici rendue visible entre virtualité et actualité, invisibilité et visibilité. Le ton fondamental univoque manifeste l'indifférence, la nondifférenciation, dans toute sa différenciation. Ce n'est pas un hasard si dans la version tardive du Couronnement d'épines le buste de Tibère s'est évanoui : toute monumentalité plastique, toute structure corporelle antiquisante et substantielle y ont en effet disparu, à l'instar de la signification chronologique du temps. Le temps n'est plus une forme de datation ou le cadre d'une narration, mais la sphère englobante de toutes les choses. Le temps chrétien consiste justement en ce que « la figure de ce monde passe ", parágei gar to schema tou kosmou toutou, praeterit enim figura huius mundi ${ }^{36}$. Ce disparaître de la figure, le chrétien doit l'avoir toujours déjà vécu pour pouvoir vivre le présent véritable, le présent du passé et de l'avenir. Cette vie se sert de la figure comme si elle ne s'en servait pas, utuntur, hoc mundo, tamquam non abutentes ${ }^{37}$. La temporalité n'est donc pas un simple déroulement linéaire mais un perpétuel dépassement dans l'éternité en comparaison de laquelle tout ce qui est déterminé n'a qu'un fondement relatif.

21 Cette relativisation, jointe à l'émergence corrélative de la temporalité, met le visible en rapport avec l'invisible. Car le fond est une virtualité pure d'où tout émerge mais qui n'est pas visible. C'est ce que le peintre rend visible. Le regard du peintre reconnaît et représente les choses par la médiation du fondement divin (göttlicher Grund). Comme dans la métaphysique de l'univocité, le premier - ou le dernier - rapport est Dieu, instance garante qui sanctifie le monde et rend possible le regard dans cette réalité sanctifiée, virtualité infinie qui permet chaque événement individuel tout en dépassant sa singularité. L'art du Titien satisfait de cette façon au paradoxe chrétien de montrer la présence de la rédemption dans un monde qui n'est pas rédempté. Le paradoxe fait référence à cette virtualité infinie qui rend lisible toute action concrète et montre en même temps l'éphémérité et le salut du monde ${ }^{38}$.

Mais ce rapport entre virtualité et actualité, qui s'accorde parfaitement à la logique théologique, est issu d'une stratégie picturale neuve. Dans son œuvre tardive en effet, le Titien élabore un rapport nouveau et réflexif entre la main et l'œil, une méthode de 
travail inédite pour l'imagination artistique. Tandis que dans le modèle du disegno, la capacité représentative conçoit en premier, l'œil jouant un rôle de médiation et la main de simple exécutrice, la main peignante entre désormais dans un rapport nouveau avec la matière, dans une sorte de créativité aveugle dont le résultat ne correspond pas à un modèle préalable et ne peut être mesuré par l'œil à un dessin antérieur. Indépendamment du fait de savoir si c'est vraiment chez le vieux Titien que cette méthode de travail est apparue pour la première fois, on pourrait parler d'une seconde invention de la peinture, tranchant clairement avec la première, où une jeune fille amoureuse reporte la silhouette de l'aimé sur la surface de la peinture pour rendre présent un absent. L'œil voit désormais ce qui est; il laisse la main au repos ou la pousse de nouveau à chercher, avec la même créativité aveugle, l'accord interne de l'œuvre qui, dorénavant, n'est plus une représentation mais une sorte de précipité permanent de ce processus de réflexion. L'autoportrait de Berlin (figure 6), où les mains du Titien sont laissées inachevées, se laisse-t-il rattacher à ce processus créatif ${ }^{39}$ Les mains donnent sans cesse à l'œil des modèles qui doivent être travaillés, elles donnent l'inachevé ; l'œil, lui, régule et dirige les mains de façon inédite.

Figure 6

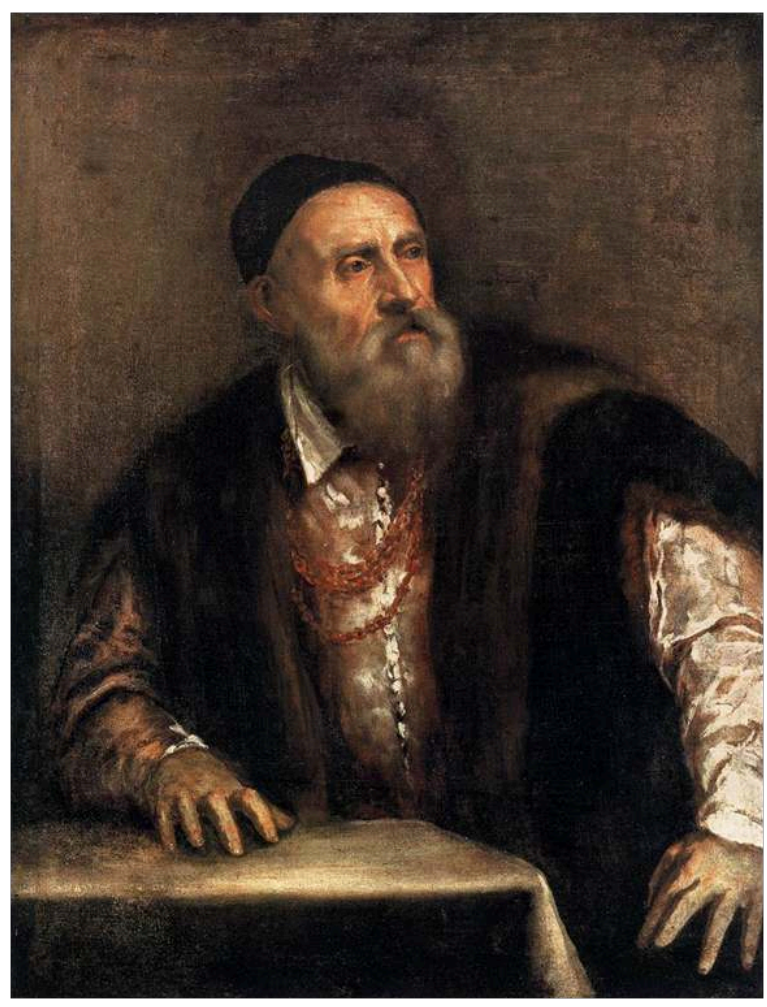

Autoportrait, Titien, 1550-1562, huile sur toile, 66 × 75 cm, Berlin, Gemäldegalerie.

Un paradoxe apparait alors, que Hans Belting a déjà souligné et que nous avons qualifié plus haut avec Luhmann de crise de l'attribution à l'époque des luttes confessionnelles $^{40}$. Car c'est précisément dans cette mobilisation de la virtualité invisible que l'image cultuelle se change en œuvre d'art. Le regard pieux du peintre qui s'est exprimé dans la vision de Dieu, dans le regard sur l'origo, commence à devenir original, ce qui a pour effet de relativiser le contenu de la représentation qui dorénavant procède de l'imagination de l'artiste. À l'époque du génie qui s'est ouverte à 
Venise au plus tard avec Giorgione, un regard autonome et de premier abord mystérieux se forme ; il ne cherche plus sa valeur dans l'objet, mais dans sa subjectivité et son pouvoir. $\mathrm{Au} \mathrm{XVI} \mathrm{X}^{\mathrm{e}}$ siècle, l'art était bel et bien devenu un pouvoir social et la différenciation $\mathrm{du}$ sous-système autonome art était irréversiblement engagée. La société avait compris «que l'art en tant que tel n'est pas un phénomène religieux ${ }^{41}$ ». Comment l'artiste, qui est toujours sous l'égide de l'action religieuse mais qui s'affirme déjà comme créateur, traite-t-il désormais l'attribution qui n'est plus déterminée ? Que signifie être un artiste ? À cette question, la carnation du Titien semble répondre en réunissant clairement deux logiques: la logique des couleurs, d'une part, où la carnation, couleur de toutes les couleurs, remplit une fonction purement picturale de vivification de la toile - du moins aux yeux de l'Européen occidental blanc-, et la logique théologique de la création qui code très généralement la structure du faire. Le peintre est tout à la fois créateur et récipiendaire de la grâce. Le lien tissé par le Titien entre la signification de la peinture et la logique de l'incarnation peut être compris comme une tentative pour formuler ce paradoxe. Et peut-être pourrait-on affirmer que même s'il a soumis sa peinture aux codes chrétiens catholiques, son traitement non figuratif des couleurs conduit à les remplir pour ainsi dire à l'excès et donc aussi à les dissoudre ${ }^{42}$. L'origine administrée théologiquement, l'origo, entre en concurrence avec l'originarité artistique, l'originalité. L'artiste doit et a le devoir d'agir souverainement, mais il lui faut en même temps le nier et nier cette négation.

\section{BIBLIOGRAPHIE}

Agamben Giorgio, Die Zeit, die bleibt. Ein Kommentar zum Römerbrief, Francfort-sur-le-Main, Suhrkamp, 2006. (Turin, 2000 pour l'édition originale en italien; pour la traduction française, voir G. Agamben, Le temps qui reste. Un commentaire de l'Épitre aux Romains, Judith Revel (trad.), Paris, Payot \& Rivages, 2004).

Aristote, De l'âme, $431 \mathrm{~b}$ 16. Pour la traduction française, voir par exemple celle de J. Tricot, Paris, Vrin, 1934 ; de Richard Bodéüs, Paris, Flammarion, 1993, de A. Jannone et E. Barbotin, Paris, Belles Lettres, 2002, de Pierre Thillet, Paris, Gallimard, 2005.

Aristote, Poétique, 1447 b 27 sq. 1448 a 1 sq. Pour la traduction française, voir par exemple celle de J. Hardy, Paris, Belles Lettres, 1965 ; de R. Dupont-Roc et J. Lallot, Paris, Seuil, 1980 ; de M. Magnien, Paris, Le Livre de Poche, 1990 ; de B. Gernez, Paris, Les Belles Lettres, 1997.

Belting Hans, Bild und Kult. Eine Geschichte des Bildes vor dem Zeitalter der Kunst, Munich, C. H. Beck, 1990. Pour la traduction française, voir H. Belting, Image et culte. Une histoire de l'image avant l'époque de l'art, Franck Muller (trad.), Paris, Éditions du Cerf, 2007.

Bohde Daniela, Haut, Fleisch und Farbe. Körperlichkeit und Materialität in den Gemälden Tizians, Emsdetten, Imorde/Berlin, Gebr. Mann, 2002.

Boschini Marco, La carta del navegar pitoresco. Dialogo tra un senator venetian deletante, e un professor de Pitura, soto nome d'Ecelanza, e de Compare [1660], Comparti in oto venti, Anna Pallucchini (ed.), Venise, 1966. 
Boulnois Olivier (éd.), Duns Scot à Paris, 1302-2002, actes du colloque de Paris, 2-4 septembre 2002, Turnhout, Brepols, 2004.

Deleuze Gilles, Différence et répétition, Paris, PUF, 1968.

D’Elia Una Roman, The Poetics of Titian's Religious Paintings, Cambridge, Cambridge University Press, 2005.

Dittman Lorenz, "Über das Verhältnis von Zeitstruktur und Farbgestaltung in Werken der Malerei”, in Festschrift Wolfgang Braunfels, Friedrich Piel et Jörg Traeger (ed), Tübingen, Wasmuth.

Florenskij Pavel, Die Ikonostase. Urbild und Grenzerlebnis im revolutionären Rußland, Stuttgart, Urachhaus, 1988.

Ferino-Padgen Sylvia, Late Titian and the Sensuality of Painting, catalogue de l'exposition, Vienne, Kunsthistorisches Museum / Venise, Accademia (2007-2008), Venise, 2007.

Funkenstein Amos, Theology and Scientific Imagination from the Middle Ages to the Seventeenth Century, Princeton, Princeton University Press, 1986.

Hetzer Theodor, "Das deutsche Element in der italienischen Malerei des sechzehnten Jahrhunderts" [1929], in Das Ornamentale und die Gestalt, Stuttgart, Urachhaus, 1987.

Hetzer Theodor, "Tizian - Geschichte seiner Farbe" [1935], in Theodor Hetzer, Schriften in neun Bänden, Gertrude Berthold (éd.), Stuttgart, Urachhaus, 1992, vol. 7.

Honnefelder Ludger, Scientia transcendens. Die formale Bestimmtheit der Seiendheit und Realität in der Metaphysik des Mittelalters und der Neuzeit (Duns Scotus - Suàrez - Wolff - Kant - Peirce), Hambourg, Meiner, 1990.

Honnefelder Ludger, Johannes Duns Scotus, Munich, C. H. Beck, 2005.

Kessler Eckard, “The intellective soul”, in Charles Schmitt, Quentin Skinner, Eckard Kessler, Jill Kraye (ed.), The Cambridge History of Renaissance Philosophy, Cambridge, Cambridge University Press, 1988.

Kleinschmidt Erich, Die Entdeckung der Intensität. Geschichte einer Denkfigur im 18. Jahrhundert, Göttingen, Wallstein Verlag, 2004.

Kruse Christiane, "Fleisch werden - Fleisch malen: Malerei als 'incarnazione'. Mediale Verfahren des Bildwerdens im Libro dell'Arte von Cennino Cennini", in Zeitschrift für Kunstgeschichte 63, 2000 .

Lohr Charles, "The Sixteenth-Century Transformation of the Aristotelian Natural Philosophy", in Eckard Keßler, Charles Lohr et Walter Sparn (ed.), Aristotelismus und Renaissance. In memoriam Charles B. Schmitt, Wiesbaden, Harrassowitz, 1988.

Luhmann Niklas, "Frühneuzeitliche Anthropologie: Theorietechnische Lösungen für ein Evolutionsproblem der Gesellschaft", in N. Luhmann, Gesellschaftsstruktur und Semantik, Studien zur Wissenssoziologie der modernen Gesellschaft, vol. 1, Francfort-sur-le-Main, Suhrkamp, 1980.

Luhmann Niklas, Die Kunst der Gesellschaft, Francfort-sur le Main, Suhrkamp, 1998.

Mâle Émile, L'art religieux de la fin du XVI $I^{e}$ siècle, du XVII siècle et du XVIII siècle. Étude sur l'iconographie après le Concile de Trente, Italie-France-Espagne-Flandres [1948], Paris, Armand Colin, 1972.

Oberthaler Elke, "Titians late style as seen in the Nymph and Shepherd", in Sylvia Ferino-Padgen (ed.), Late Titian and the Sensuality of Painting, catalogue de l'exposition, Vienne, Kunsthistorisches Museum / Venise, Accademia (2007-2008), Venise, 2007. 
Ockham Guillaume (d'), Texte zur Theorie der Erkenntnis und der Wissenschaft, Rudi Imbach (ed.), Stuttgart, Reclam, 1984.

Panofsky Erwin, “Die Perspektive als ‘symbolische Form”, in E. Panofsky, Aufsätze zu Grundfragen der Kunstwissenschaft, Hariolf Oberer et Egon Verheyen (ed.), Berlin, B. Hessling Verlag, 1964. Traduction française sous la direction de Guy Ballangé, E. Panofsky, « La perspective comme forme symbolique », in E. Panofsky, La perspective comme forme symbolique et autres essais, Paris, Minuit, 1975.

Panofsky Erwin, Problems in Titian. Mostly Iconographic, New York, New York University Press, 1969 [posthume]. Traduction française par J. Hazan et A. Delord, E. Panofsky, Le Titien. Questions d'iconographie, Paris, Hazan, 1989.

Paul, Épitre aux Corinthiens 7, 31.

Pedrocco Filippo, Tizian, Munich, Hirmer, 2000.

Pomponazzi Pietro, Abhandlung über die Unsterblichkeit der Seele, latin et allemand, traduit et édité par Burkhard Mojsisch, Hambourg, Meiner Verlag, 1990.

Poppi Antonio, La Filosofia nelle Studio Francescano del Santo a Padova, Padoue, Centro Studi Antoniani, 1989.

Poppi Antonio, Introduzione all'aristotelismo padovano, Padoue, Antenore, 1991.

Riegl Alö̈s, Spätrömische Kunstindustrie [1901], Darmstadt, Wissensch. Buchgesellschaft, 1973 (reproduction photomécanique de la $2^{\mathrm{e}}$ édition, Vienne, Österreichische Staatsdruckerei, 1927).

Rombach Heinrich, Substanz, System, Struktur. Die Ontologie des Funktionalismus und der philosophische Hintergrund der modernen Wissenschaft, 2 vol., Fribourg/Munich, Alber, 1981.

Rombach Heinrich, "Die Welt des Barock. Versuch einer Strukturanalyse”, in Kovacs Feuchtmüller (ed.), Welt des Barock, Vienne/Fribourg/Bâle, 1986.

Rosand David, Titien. «L'art plus fort que la nature », Paris, Gallimard, 1993. (1978 pour l'édition originale américaine).

Rosand David, Painting in Sixteenth-Century Venice, Titian, Veronese, Tintoretto, Cambridge, Cambridge University Press, 1997.

Rosen Valeska (von), Mimesis und Selbstbezüglichkeit in Werken Tizians, Emsdetten, Imorle/Berlin, Gebr. Mann Verlag, 2001.

Sandrart Joachim (von), Teutsche Academie der Bau-, Bild- und Mahlerey-Künste, Nuremberg, 1675, réimpression Nördlingen, 1995.

Schelling Friedrich Wilhelm Joseph, Philosophie der Kunst, in Sämtliche Werke, Karl Friedrich August Schelling (ed.), vol. 5, Stuttgart et Augsburg, J. G. Cotta, 1859.

Schulthess Peter, Imbach Rudi, Die Philosophie im lateinischen Mittelalter. Ein Handbuch mit einem biobibliographischen Repertorium, Zurich / Düsseldorf, Artemis \& Winckler, 1996.

Simon Gérard, Le regard, l'être et l'apparence dans l'optique de l'Antiquité, Paris, Seuil, 1988.

Wandel Lee Palmer, "The Reformation and the visual arts", in Po-Chia Hsia (ed.), The Cambridge History of Christianity, vol. 6. Reform and Expansion 1500-1660, Cambridge, Cambrige University Press, 2007.

Warnke Martin, "Nah und Fern zum Bilde", in M. Warnke, Nah und Fern zum Bilde. Beiträge zu Kunst und Kunsttheorie, Michael Diers (ed), Cologne, DuMont, 1997. 
Weber Gregor J. M., Weddigen Tristan, "Tizians Bildnis des Farbenhändlers Alvise dalla Scala”, in Andreas Henning, Uta Neidhardt et Martin Roth (ed.), "Man könnt vom Paradies nicht angenehmer träumen”. Festschrift für Prof. Dr. Harald Marx, Berlin et Munich, Imorde, 2009.

Wilde Johannes, De Bellini à Titien. Texture, forme, couleur dans l'art vénitien, Paris, Macula, 1993. (Oxford, 1981 pour l'édition originale en anglais).

\section{NOTES}

1. Friedrich Wilhelm Joseph Schelling, Philosophie der Kunst, in Sämtliche Werke, K. F. A. Schelling (ed.), vol. 5, Stuttgart et Augsburg, J. G. Cotta, 1859, p. 540.

2. «Même quand elle considère des problèmes de contingence, la religion déduit [...] de la société les problèmes d'imputation correspondants pour les rapporter au dieu qui a créé le monde et le conserve. La "nature créée" symbolise donc toujours aussi les limites de ce qui peut être imputé à l'homme. [...] Il en résulte que les responsabilités qui doivent être socialement régulées sont également maintenues dans certaines limites. Elles demeurent dans le cadre des exigences de conformité. [...] C'est cette fonction de délestage des problèmes d'attribution que le conflit confessionnel mettra à mal ; dans un monde toujours plus complexe, elle ne sera plus exercée par la religion » (Niklas Luhmann, "Frühneuzeitliche Anthropologie: Theorietechnische Lösungen für ein Evolutionsproblem der Gesellschaft", in N. Luhmann, Gesellschaftsstruktur und Semantik, Studien zur Wissenssoziologie der modernen Gesellschaft, vol. 1, Francfort-sur-le-Main, Suhrkamp, p. 162-234, p. 175). Sauf mention contraire, les citations sont traduites par Audrey Rieber.

3. Pavel Florenskij, Die Ikonostase. Urbild und Grenzerlebnis im revolutionären Rußland, Stuttgart, Urachhaus, 1988, p. 75. Les textes de Florenskij contiennent toujours une critique véhémente du subjectivisme de la peinture occidentale qui se forme aussi dans les moyens formels de la peinture.

4. Voir Filippo Pedrocco, Tizian, Munich, Hirmer, 2000, p. 175 et p. 294. On sait que le Tintoret avait acquis le tableau qui, après sa mort, passa aux mains du prince électeur de Bavière. Celui-ci le fit d'abord accrocher dans le château de Schleissheim. Sur l'histoire des deux œuvres, voir les indications exhaustives de Valeska von Rosen, Mimesis und Selbstbezüglichkeit in Werken Tizians, Emsdetten, Imorde/Berlin, Gebr. Mann Verlag, 2001, p. 205 sq. et p. 367 sq.

5. Ibid., p. 398-405. Il faut supposer que de nombreuses images ont été copiées et conservées dans l'atelier du Titien avant d'être livrées. Elles connaissaient donc parfois le sort d'être à nouveau travaillées par le Titien.

6. Voir Una Roman D'Elia, The Poetics of Titian's Religious Paintings, Cambridge, Cambridge University Press, 2005, p. 43.

7. Voir Theodor Hetzer, "Tizian - Geschichte seiner Farbe" [1935], in Theodor Hetzer, Schriften in neun Bänden, Gertrud Bertold (ed.), Stuttgart, Urachhaus, 1992, vol. 7, p. 39-305, p. 175 sq. Sur la méthode de travail du vieux Titien, voir Elke Oberthaler, "Titians late style as seen in the Nymph and Shepherd", in Sylvia Ferino-Padgen (ed.), Late Titian and the Sensuality of Painting, catalogue d'exposition, Vienne, Kunsthistorisches Museum / Venise, Accademia (2007-2008), Venise, 2007, p. 113-123, p. 117 : He « certainly did not paint individual pictorial or color areas one after the other, but rather placed accents with the brush almost simultaneously over the entire painting, and so worked out his composition, revised and further developed following interruptions, always considering the entire picture surface». Voir Valeska von Rosen, Mimesis und Selbstbezüglichkeit, op. cit., p. 386-389. Ce style tardif du Titien a presque été unanimement rejeté par ses contemporains. Voir Rosen (note 4), p. 390-398. Même dans l'atelier du Titien, son travail était saboté. «On tenait dans l'ensemble sa première manière pour la meilleure ; mais lui la renia dans son grand âge / et gâcha des morceaux de différente qualité / qu'il avait réalisés dans sa 
jeunesse / en les modifiant / dans sa vieillesse : c'est pourquoi les siens / dès qu'il voulait modifier une telle œuvre / mélangeaient de l'huile d'olive avec les couleurs / pour que les couleurs ne sèchent pas / et qu'on puisse les essuyer en l'absence du Titien, ce qui permit de conserver certaines bonnes pièces " (Joachim Sandrart, Teutsche Academie der Bau-, Bild- und Mahlerey-Künste, Nuremberg, 1675, réimpression Nördlingen, 1995, p. 165, cité par Rosen, p. 391). 8. Voir sur ce point Lorenz Dittman, "Über das Verhältnis von Zeitstruktur und Farbgestaltung in Werken der Malerei”, in Festschrift Wolfgang Braunfels, Friedrich Piel et Jörg Traeger (ed), Tübingen, Wasmuth, 1977, p. 93-103, p. 102 sq.

9. Hetzer analyse ce revirement dans la composition et la conception des corps à partir de deux images de Danaé : celle de Naples, de 1545, et celle de Madrid, de 1554. Voir Hetzer, Tizian, op. cit. (note 7), p. 156 sq.

10. «La beauté et la détermination des objets doivent être détruites pour que l'unité et la beauté de la conception du génie l'emportent ", commente Hetzer dans son Tizian, op. cit. (note 7), p. 158. Pour l'analyse de cette immobilisation sous l'angle de la technique picturale, voir Rosen (note 4), p. 386 sq.

11. "E veramente [...] egli con ragione cosi operò ; perché, volendo imitare l'operazione del Sommo Creatore, [...] nel formar questo corpo umano, lo formò di terra con le mani", commente Marco Boschini dans La carta del navegar pitoresco. Dialogo tra un senator venetian deletante, e un professor de Pitura, soto nome d'Ecelanza, e de Compare [1660], Comparti in oto venti, édité et commenté par Anna Pallucchini, Venise, 1966, p. 711 sq., cité par Rosen, op. cit. (note 4), p. 416 sq. Sur la carnation chez le Titien, voir Daniela Bohde, Haut, Fleisch und Farbe. Körperlichkeit und Materialität in den Gemälden Tizians, Emsdetten, Imorde / Berlin, Gebr. Mann, 2002, p. 59 par exemple : «C'est la question autour de laquelle les critiques d'art du XvI ${ }^{\mathrm{e}}$ siècle tournent toujours à nouveau : dans les tableaux du Titien, la couleur devient chair. [...] Le Titien se place ainsi dans une tradition qui apparaît dès le traité de peinture de Cennino Cennini. Comme l'a montré Christiane Kruse, le concept théologique d'“incarnation" apparaît dans le contexte de la technique picturale avec le "Libro dell'arte" de Cennini. Le processus du devenir image y est décrit comme incarnare et identifié au colorire. D'un côté, le concept est utilisé de manière concrète pour désigner la couleur de la chair, de l'autre, il indique la capacité de la peinture à incarner ce qui n'est pas visible ». Bohde se réfère à l'analyse par Christiane Kruse du traité de Cennini. C'est dans cet ouvrage que, au début $d u x^{\mathrm{e}}$ siècle, est décrite pour la première fois une technique de peindre, en l'occurrence celle de Giotto, «dont l'intention est l'imitation illusionniste de la nature " ("Fleisch werden - Fleisch malen: Malerei als 'incarnazione'. Mediale Verfahren des Bildwerdens im Libro dell'Arte von Cennino Cennini”, in Zeitschrift für Kunstgeschichte, 63, 2000, p.305-325, p.317). En rupture avec les idées médiévales, le Libro esquisse une théorie des médias «qui, avec le concept d'incarnazione, décrit le processus du “devenir image" d'un homme sur le mur » - c'est de l'art de la fresque qu'il s'agit (Ibid., p. 306). Cennini entend par incarnazione aussi bien l'incarnation de concepts, c'est-à-dire des dessins, que la représentation de corps humains vivants. «La creatio ainsi comprise complète la création divine dans la mesure où elle recrée dans le médium de l'image ce qu'au commencement du monde, Dieu a initié une unique fois » (p. 323). Lee Palmer Wandel fait remarquer la signification particulière de l'incarnation pour la production d'images catholique après la Réforme: "The Catholic Church, in keeping with its understanding of the operation of grace, took up images as important in a massive project of proselytization and conversion - images could touch the spirit, could render in matter the multiplex meaning of the Incarnation for humanity. Luther, with his radical emphasis on faith, gave images the least weight: in the tradition that looked to him as a founder, images remained, but they existed apart from the great drama of Incarnation. » (L. P. Wandel, "The Reformation and the visual arts", in Po-Chia Hsia (ed.), The Cambridge History of Christianity, vol. 6. Reform and Expansion 1500-1660, Cambridge, Cambrige University Press, 2007, p. $345-371$. p. 369. 
12. Je renvoie sur ce point à Martin Warnke, "Nah und Fern zum Bilde", in M. Warnke, Nah und Fern zum Bilde. Beiträge zu Kunst und Kunsttheorie, Michael Diers (ed), Cologne, DuMont, 1997, p. 6-15. On consultera aussi avec profit les articles consacrés à la technique de travail du vieux Titien dans le catalogue d'exposition édité par Sylvia Ferino-Padgen, Late Titian and the Sensuality of Painting, op. cit. (note 7). On trouve chez le vieux Titien des morceaux qui nous bluffent littéralement par leur modernité, tel par exemple le coin supérieur gauche du tardif Garçon avec deux chiens conservé à Madrid (Prado), où l'on ne voit plus que des taches blanches et bleues qui ne prennent une qualité compositionnelle qu'à une certaine distance.

13. Voir Filippo Pedrocco, Tizian, Munich, Hirmer, 2000, p. 233.

14. Erwin Panofsky, Problems in Titian. Mostly Iconographic, New York, New York University Press, 1969 [posthume], cité d'après F. Pedrocco, Tizian, op. cit., p. 233. Il existe une traduction française de ce texte par J. Hazan et A. Delord : E. Panofsky, Le Titien. Questions d'iconographie, Paris, Hazan, 1989.

15. Filippo Pedrocco, Tizian, op. cit., p. 236.

16. Voir Gregor J. M. Weber et Tristan Weddigen, "Tizians Bildnis des Farbenhändlers Alvise dalla Scala", in Andreas Henning, Uta Neidhardt et Martin Roth (ed.), "Man könnt vom Paradies nicht angenehmer träumen". Festschrift für Prof. Dr. Harald Marx, Berlin et Munich, Imorde, 2009, p. 58-65.

17. Theodor Hetzer, "Das deutsche Element in der italienischen Malerei des sechzehnten Jahrhunderts“ (1929), in Das Ornamentale und die Gestalt, Stuttgart, Urachhaus, 1987, p. 113.

18. Ibid., p. 112.

19. Th. Hetzer, Tizian (note 7), p. 92. Voir aussi l'analyse similaire de Panofsky : «À ses débuts il considère encore la couleur comme un élément adhérent aux objets représentés ; dans sa période centrale il l'appréhende comme un élément essentiellement déterminé par la structure chromatique d'ensemble apparaissant sur le panneau ou sur la toile de telle sorte que nous pouvons parler de "couleur-tableau" et non plus de "couleur-objet". Dans la phase finale enfin, il en arrive à considérer la couleur comme quelque chose de diffus dans l'espace ("couleur-espace" pourrait-on dire) et qui peut simplement se concentrer et se diversifier dans des zones particulières. » Le Titien. Questions d'iconographie, op. cit., p. 29 sq. Voir aussi Johannes Wilde, De Bellini à Titien. Texture, forme, couleur dans l'art vénitien, Paris, Macula, 1993, p. 232 (Oxford, 1981 pour l'édition originale en anglais) : «La couleur semble être l'unique substance de cette réalité, sublime et pathétique à la fois ». Malgré sa critique de la méthode " perceptive » de Hetzer, dont il considère qu'elle ne prend pas suffisamment en compte les éléments iconologiques et les circonstances historiques (voir David Rosand, Painting in Sixteenth-Century Venice, Titian, Veronese, Tintoretto, Cambridge, Cambridge University Press, 1997, p. 63), Rosand déclare de la même façon que « le grain de la toile tout comme les effets de matière de la surface picturale rugueuse constituent la substance quasi vivante qui remplace, sans l'imiter, celle de la réalité : la chair, les tissus, la pierre. Des zones de lumière surgissent du fond obscur. Il n'y a guère de volumes consistants dans cet univers pétri de couleurs» (David Rosand, Titien, «L'art plus fort que la nature », Paris, Gallimard, 1993, p. 118 (1978 pour l'édition originale américaine).

20. Th. Hetzer, Tizian, op. cit. (note 7), p. 162.

21. Amos Funkenstein a montré que c'est justement l'idée théologique de l'omniprésence divine qui constitue l'arrière-fond de ce changement radical dans les sciences. Voir A. Funkenstein, Theology and Scientific Imagination from the Middle Ages to the Seventeenth Century, Princeton, Princeton University Press, 1986. Le corps mondain prend alors les propriétés de ce corps idéal. Heinrich Rombach qualifie la philosophie moderne en général d'ontologie du champ; il étudie ses élaborations successives, du Cusain à Leibniz (H. Rombach, Substanz, System, Struktur. Die Ontologie des Funktionalismus und der philosophische Hintergrund der modernen Wissenschaft, 2 vol., Fribourg/Munich, Alber, 1981 et, du même auteur, "Die Welt des Barock. Versuch einer 
Strukturanalyse", in Kovacs Feuchtmüller (ed.), Welt des Barock, Vienne/Fribourg/Bâle, 1986, p. 9-23.

22. Voir Aristote, De l'âme, 431 b 16.

23. Erwin Panofsky, "Die Perspektive als 'symbolische Form”, in E. Panofsky, Aufsätze zu Grundfragen der Kunstwissenschaft, Hariolf Oberer et Egon Verheyen (ed.), Berlin, B. Hessling Verlag, 1964, p. 99-168, et tout particulièrement p. 108-111. Pour la traduction française sous la direction de Guy Ballangé, voir E. Panofsky, "La perspective comme forme symbolique », in E. Panofsky, La perspective comme forme symbolique et autres essais, Paris, Minuit, 1975. Voir aussi sur ce thème les analyses d'Alois Riegl sur les débuts d'un espace unifié dans l'art de l'Antiquité tardive : A. Riegl, Spätrömische Kunstindustrie [1901], Darmstadt, Wissensch. Buchgesellschaft, 1973 (reproduction photomécanique de la $2^{\mathrm{e}}$ édition, Vienne, Österreichische Staatsdruckerei, 1927), p. 398-405.

24. Voir par exemple Gérard Simon, Le regard, l'être et l'apparence dans l'optique de l'Antiquité, Paris, Seuil, 1988.

25. Voir Aristote, De anima, 417 b 32 sq. (pour la traduction française du De l'âme, voir par exemple celle de J. Tricot, Paris, Vrin, 1934 ; de Richard Bodéüs, Paris, Flammarion, 1993, de A. Jannone et E. Barbotin, Paris, Belles Lettres 2002, de Pierre Thillet, Paris, Gallimard, 2005) ; Poétique, 1447 b 27 sq. 1448 a 1 sq. (Pour la traduction française de La poétique, voir par exemple celle de J. Hardy, Paris, Belles Lettres, 1965 ; de R. Dupont-Roc et J. Lallot, Paris, Seuil, 1980 ; de M. Magnien, Paris, Le Livre de Poche, 1990 ; de B. Gernez, Paris, Les Belles Lettres, 1997). Aristote pressent une fois le phénomène esthétique moderne lorsqu'il observe de manière remarquable que lorsqu'on n'a encore jamais vu un objet, le schéma cognitif est supprimé et que le plaisir alors éprouvé relève de toute évidence de l'exécution, des couleurs, etc., bref de propriétés purement esthétiques (Poétique, 1448 b 17 sq.)

26. Voir par exemple le commentaire de la physique, prologue $n^{\circ} 17$ (Guillaume d'Ockham, Texte zur Theorie der Erkenntnis und der Wissenschaft, Rudi Imbach (ed.), Stuttgart, Reclam, 1984, p. 196 sq.

27. Voir Gilles Deleuze, Différence et répétition, Paris, PUF, 1968, chapitre 1.

28. Voir Ludger Honnefelder, Scientia transcendens. Die formale Bestimmtheit der Seiendheit und Realität in der Metaphysik des Mittelalters und der Neuzeit (Duns Scotus - Suàrez - Wolff - Kant - Peirce), Hambourg, Meiner, 1990 et, du même auteur, Johannes Duns Scotus, Munich, C. H. Beck, 2005, p. 59-62 et passim. Le lecteur français consultera aussi avec profit O. Boulnois (ed.), Duns Scot à Paris, 1302-2002, actes du colloque de Paris, 2-4 septembre 2002, Turnhout, Brepols, 2004.

29. Voir Heinrich Rombach, Substanz, System, Struktur. Die Ontologie des Funktionalismus und der philosophische Hintergrund der modernen Wissenschaft, op. cit. (note 21) et, du même auteur, "Die Welt des Barock. Versuch einer Strukturanalyse", op. cit. (note 21), p. 13.

30. On trouve de nombreux passages de ce genre chez Erich Kleinschmidt, Die Entdeckung der Intensität. Geschichte einer Denkfigur im 18. Jahrhundert, Göttingen, Wallstein Verlag, 2004, p. 15-25.

31. Valeska von Rosen, Mimesis und Selbstbezüglichkeit in Werken Tizians, op. cit., p. 430 et p. 422-430. 32. Voir Peter Schulthess et Rudi Imbach, Die Philosophie im lateinischen Mittelalter. Ein Handbuch mit einem bio-bibliographischen Repertorium, Zurich/Dusseldorf, Artemis \& Winckler, 1996, p. 232 pour cette citation.

33. Antonio Poppi, Introduzione all'aristotelismo padovano, Padoue, Antenore, 1991, p. 18 et, du même auteur, La Filosofia nelle Studio Francescano del Santo a Padova, Padoue, Centro Studi Antoniani, 1989.

34. Voir Charles Lohr, "The Sixteenth-Century Transformation of the Aristotelian Natural Philosophy", in Eckard Keßler, Charles Lohr et Walter Sparn (ed.), Aristotelismus und Renaissance. In memoriam Charles B. Schmitt, Wiesbaden, Harrassowitz, 1988, p. 89-99, et tout particulièrement p. 96-99. Tout bien considéré, on est donc face à une situation où tout le monde travaille au concept aristotélicien de substance - n'oublions pas qu'on est avant Galilée et Bruno - ce qui pose 
la question de savoir si et dans quelle mesure ces débats ont trouvé un écho dans la réflexion artistique voire, plus directement encore, chez le Titien.

35. N. Luhmann, "Frühneuzeitliche Anthropologie: Theorietechnische Lösungen für ein Evolutionsproblem der Gesellschaft", in Gesellschaftsstruktur und Semantik, op. cit. (note 2), p. 197.

36. Paul, Épitre aux Corinthiens, 7, 31. Voir Giorgio Agamben, Die Zeit, die bleibt. Ein Kommentar zum Römerbrief, Francfort-sur-le-Main, Suhrkamp, 2006, p. 46 sq. (Turin, 2000 pour l'édition originale en italien; pour la traduction française, voir G. Agamben, Le temps qui reste. Un commentaire de l'Épitre aux Romains, traduction de Judith Revel, Paris, Payot \& Rivages, 2004).

37. « et ceux qui usent de ce monde, comme n'en usant pas », loc. cit.

38. Je ne développerai pas davantage les références si fréquentes du Titien au temps qui passe, au sens du memento mori. Celui-ci est en quelque sorte la forme négative du temps du Salut, le temps terrestre sans rapport à Dieu. Voir sur cette question les analyses exhaustives d'Erwin Panofsky, Le Titien. Questions d'iconologie, op. cit., chapitre 4 : « Réflexions sur le temps ».

39. Cette hypothèse a été émise par Andreas Beyer.

40. Hans Belting, Bild und Kult. Eine Geschichte des Bildes vor dem Zeitalter der Kunst, Munich, C. H. Beck, 1990, p. 525 sq. et tout particulièrement p. 537 sq. Pour la traduction française, voir H. Belting, Image et culte. Une histoire de l'image avant l'époque de l'art, Franck Muller (trad.), Paris, Éditions du Cerf, 2007.

41. «Et c'est de là justement que surgit le problème de savoir si une sorte d'art et laquelle convient à l'office religieux; le protestantisme et l'église catholique trouvèrent des réponses différentes à ce problème ", Niklas Luhmann, Die Kunst der Gesellschaft, Francfort-sur le Main, Suhrkamp, 1998, p. 297 sq.

42. Évidemment, le rapport de double codification devient surtout conscient lorsqu'il ne fonctionne plus. Pomponazzi par exemple avait utilisé un concept de double vérité pour pouvoir exprimer sans danger ses thèses contre l'immortalité de l'âme. Mais cela n'a pas empêché son livre d'être brûlé en public à Venise, en 1516. Voir Pietro Pomponazzi, Abhandlung über die Unsterblichkeit der Seele, latin et allemand, traduit et édité par Burkhard Mojsisch, Hambourg, Meiner Verlag, 1990, p. 52 sq. et chap. 15, p. 228 sq. Voir aussi l'introduction de Mojsisch, p. VIII et Eckard Kessler, "The intellective soul”, in Charles Schmitt, Quentin Skinner, Eckard Kessler et Jill Kraye (ed.), The Cambridge History of Renaissance Philosophy, Cambridge, Cambrige University Press, 1988, p. 484-534, p. 500-507. Ici toutefois le modèle a échoué plus rapidement que dans l'art, une différence qui était déjà repérable immédiatement après Pomponazzi, voir Kessler, op. cit., p. 507 sq. En 1573, Véronèse sera convoqué devant l'inquisition vénitienne pour avoir donné une image trop profane de la cène, plus précisément pour avoir introduit dans sa peinture des « figures indignes ». L'art baroque n'est pas l'exécutant de l'église, car celle-ci « fit mieux que de surveiller, de blâmer, d'interdire, elle inspira » (Émile Mâle, L'art religieux de la fin du XvI siècle, du XVII siècle et $d u X^{X V I I}{ }^{e}$ siècle. Étude sur l'iconographie après le Concile de Trente, Italie-France-EspagneFlandres, [1948], Paris, Armand Colin, 1972, p. 511 ; de son côté, « l'art n'avait encore rien tenté de pareil ; il arrivait là, lui aussi, à l'extrême limite du possible » (Ibid.) et il faudrait étudier ici dans le détail les formes de synthèse, de conflit et de dissolution. 


\section{RÉSUMÉS}

Logique picturale et logique religieuse se rencontrent de façon remarquable dans l'œuvre tardive du Titien puisque à la critique de la philosophie substantialiste aristotélicienne par une métaphysique de l'autodifférenciation (Jan Duns Scot) semble correspondre l'abandon de la théorie du disegno (Vasari) et d'une conception de la couleur comme accident des choses au profit d'une pratique de la couleur comme autodifférenciation d'une teinte fondamentale homogène, la carnation. Loin de vouloir réduire ce parallèle à une question d'influences ou d'esprit du temps, il s'agira d'analyser, à partir des deux versions du Couronnement d'épines du Titien, cette nouvelle conception de l'œuvre et de la création que la coloristique traduit tout particulièrement.

\section{INDEX}

Personnes citées : Titien, Aristote, Duns Scot (Jan)

Mots-clés : autodifférenciation, couleur, création, forme, incarnation, matière, œuvre, relation, substance

\section{AUTEURS}

\section{THOMAS KISSER}

Philosophe, Thomas Kisser est membre du Studiengang « Aisthesis » (Munich), membre de la Spinoza Gesellschaft et de la commission d'édition des œuvres de Schelling. Il a participé, en 2000, à l'édition du tome 6 des œuvres complètes et, en 2010, à celle d'un volume de lettres (Briefe, 1800-1802) (voir Historisch-kritische Ausgabe. Im Auftrag der Schelling-Kommission der Bayerischen Akademie der Wissenschaften). On lui doit également : avec M. Czelinski, R. Schnepf, M. Senn et J. Stenzel, Transformation der Metaphysik in die Moderne: Zur Gegenwärtigkeit der theoretischen und praktischen Philosopie Spinozas, 2003 ; avec J. Jantzen et H. Traub, Grundlegung und Kritik. Der Briefwechsel zwischen Schelling und Fichte 1794-1802, 2005 ; avec M. Bollacher et M. Walther, Ein neuer Blick auf die Welt: Spinoza in Literatur, Kunst und Ästhetik, 2010 ; avec M. Tiedtke et D. Rippl, Angst. Dimensionen eines Gefühls, 2011 ; Selbstbewußtsein und Interaktion. Spinozas Theorie der Individualität, 1998. Il a également dirigé un cahier spécial des Studia Leibnitiana: Metaphysik und Methode. Descartes, Spinoza, Leibniz im Vergleich, 2010, ainsi qu'un ouvrage collectif : Bild und Zeit, 2010. 